\title{
A short-term comparison of two methods of sputum expectoration in cystic fibrosis
}

\author{
K. Chatham, A.A. Ionescu, L.S. Nixon, D.J. Shale
}

A short-term comparison of two methods of sputum expectoration in cystic fibrosis. K. Chatham, A.A. Ionescu, L.S. Nixon, D.J. Shale. (C) ERS Journals Ltd 2004. ABSTRACT: The aim of this study was to determine whether repeated maximum inspiratory vital capacity manoeuvres against a fixed resistance increased effective short-term sputum clearance in adults with cystic fibrosis (CF).

Twenty adults with $C F$ were randomised to receive, on alternate days, either standardised physiotherapy (SP) for $30 \mathrm{~min}$, comprising postural drainage and the active cycle of breathing technique, or a series of resistive inspiratory manoeuvres (RIM) at $80 \%$ of their maximum sustained inspiratory pressure developed between residual volume and total lung capacity during the first 4 days of the treatment of an exacerbation of respiratory symptoms. Expectorated sputum was collected during and for $30 \mathrm{~min}$ after each treatment and weighed. Total protein, immunoreactive interleukin (IL)-8 and human neutrophil elastase (HNE) concentrations, and the amount of each component expectorated, were determined.

Compared with SP, RIM increased sputum weight two-fold, independent of treatment order or day. The concentrations of protein, IL-8 and HNE in sputum were similar for both treatments, while the quantity expectorated was greater with RIM treatment.

In conclusion, short-term resistive inspiratory manoeuvres treatment was more effective at clearing sputum and inflammatory mediators than standardised physiotherapy. Eur Respir J 2004; 23: 435-439.
Dept of Physiotherapy and Section of Respiratory and Communicable Diseases, University of Wales College of Medicine, Academic Centre, Llandough Hospital, Cardiff and Vale NHS Hospital Trust, Penarth, UK.

Correspondence: D.J. Shale, Section of Respiratory and Communicable Diseases, University of Wales College of Medicine, Academic Centre, Cardiff and Vale NHS Trust, Llandough Hospital, Penarth, Vale of Glamorgan, CF64 2XX, UK.

Fax: 442920716416

E-mail: shaledj@cardiff.ac.uk

Keywords: Cystic fibrosis, physiotherapy, resistive inspiratory manoeuvres

Received: July 222003

Accepted after revision: November 262003

A.A. Ionescu and L.S. Nixon were supported by CF Trust UK project grants. Other support was from the Astra Foundation $\mathrm{UK}$ and GlaxoSmithKline UK.
In cystic fibrosis (CF) airways secretions are thick, tenacious and difficult to clear from the respiratory system, particularly during periods of exacerbation of respiratory symptoms. Abnormalities of airway secretions are due to the effects of altered CF transmembrane receptor function, and include alterations in ion transport and hypersecretion of modified mucus [1-3]. Secondary to this are the effects of chronic infection and the host inflammatory response, which leads to airways secretions rich in viable bacteria and their exoproducts, host-derived neutrophils, plasma proteins, deoxyribonucleic acid, tissue damaging enzymes and proinflammatory cytokines $[1,4,5]$. This process occurs from early in life and becomes continuous in the majority of patients, leading to chronic sputum production [6]. Progressive parenchymal lung injury leads to airway instability, promoting hyperinflation, dynamic collapse and impaired clearance of sputum [3-6]. Interleukin (IL)-8, a major neutrophil chemotactic cytokine, is increased in sputum and bronchoalveolar lavage obtained from patients with $\mathrm{CF}$, and the concentration is related to clinical severity and lung function [7-9]. The sputum levels of IL-8 and neutrophil elastase have been used as indicators of local inflammation in $\mathrm{CF}$, asthma and COPD [8-11].

Chest physiotherapy is an integral component of the treatment plan of patients with CF [12-15]. A variety of treatment approaches have been developed with the common goal of maximising sputum clearance, which it is believed will reduce sputum retention, atelectasis and the duration of exposure to injurious oxidant and proteolytic agents produced by the host inflammatory response. Traditionally, physiotherapy has consisted of postural drainage with additional percussive, vibratory or expiratory manoeuvres to mobilise sputum from peripheral airways to larger central airways for clearance by coughing. However, there is no consensus as to which approach is the most effective, although there are advocates of different therapeutic regimens using various techniques and devices to attain effective expectoration [13-18]. A metaanalysis of 35 studies concluded that standardised physiotherapy (SP) enhanced sputum clearance and improved the forced expiratory volume in one second (FEV1), and that additional methods added little to SP [15].

There is a growing population of adults with $\mathrm{CF}$, many of who find it difficult to adhere to physiotherapy regimens, with reported levels of only $40-50 \%$ adherence [19]. This occurs for a variety of reasons, including not accepting the rationale for continued treatment and the problems of fitting treatment in with domestic or workplace pressures [20]. Treatments which clear secretions with a minimum of disruption to lifestyle, but which are as effective as traditional forms of physiotherapy, may have a positive influence upon disease status due to an increased compliance with such treatments, although there is little evidence for this at present [15].

The current authors have previously described the use of a fixed-load method for assessing inspiratory muscle function, which can also be used for inspiratory muscle training [21]. Patients in such studies reported that repeated inspiratory manoeuvres against a resistance, a Muller manoeuvre, resulted in increased sputum expectoration. This effect may 
be similar to that seen in exercise, which has been associated with increased sputum clearance as a secondary effect $[13,15]$. To study this observation and its potential use in the management of patients with $\mathrm{CF}$, the effect of resistive inspiratory manoeuvres (RIM) against a fixed resistance was compared with that of supervised respiratory SP, incorporating the active cycle of breathing, on sputum clearance in adult patients chronically infected with Pseudomonas aeruginosa. In addition, the impact of these treatments on the clearance of inflammatory mediators during the first 4 days of the treatment of an exacerbation of respiratory symptoms was determined.

\section{Material and methods}

\section{Patients}

A total of 20 adult patients (10 female) with proven $\mathrm{CF}$ (sweat $\mathrm{Na}^{+}$and $\mathrm{Cl}^{-}>70 \mathrm{mmol} \cdot \mathrm{L}^{-1}$ and an appropriate genotype), with chronic pulmonary infection with $P$. aeruginosa (defined as more than six isolations from sputum in the preceding year), were studied. They were studied when presenting with an exacerbation of respiratory symptoms, defined as a combination of increased cough, shortness of breath, sputum production and a reduction in FEV $1>10 \%$ of their usual value with bacteriological confirmation of a heavy growth of $P$. aeruginosa on culture of sputum. All were admitted to hospital for intravenous antibiotic treatment and gave written informed consent to be included in the study, which had Local Research Ethics Committee approval. Participants were randomly allocated to alternate day treatment with either the RT2 inspiratory resistance device (DeVilbiss Healthcare UK Ltd, Wollaston, UK) or physiotherapy for 4 days, starting from the first day of treatment with antibiotics, coinciding with the day of admission. For each patient, treatment started with one or the other technique. Hence, both treatments were applied twice with the patient randomly allocated to receive the same treatment on days 1 and 3 or days 2 and 4 . All treatment sessions were performed under supervision and at the same time of the day. All usual medications were administered during the study days; the inhaled and/or nebulised treatments were standardised and administered before the study interventions and were the same on all study days.

\section{Interventions}

SP comprised a supervised session of 30 min including three postural drainage positions with percussion administered by the physiotherapist and use of the forced expiration technique [16]. Periods of relaxed breathing and thoracic expansion exercises were also used as described in the active cycle of breathing [14]. Patients were already familiar with, and trained in the use of, the active cycle of breathing technique and postural drainage with percussion, which comprised the SP sessions.

The inspiratory resistance protocol involved the use of the RT2 hand-held manometer with a fixed leak of $2 \mathrm{~mm}$ and attached to a lap top computer loaded with the software programme of the RT2 device. The maximum inspiratory pressure generated during a full inspiratory vital capacity from residual volume (RV) was determined three times for each patient. This was shown as a sustained maximum inspiratory pressure (MIP) (SMIP) curve on a computer screen (fig. 1). The software re-set the maximum pressure curve on screen at $80 \%$ of the original and the patients then

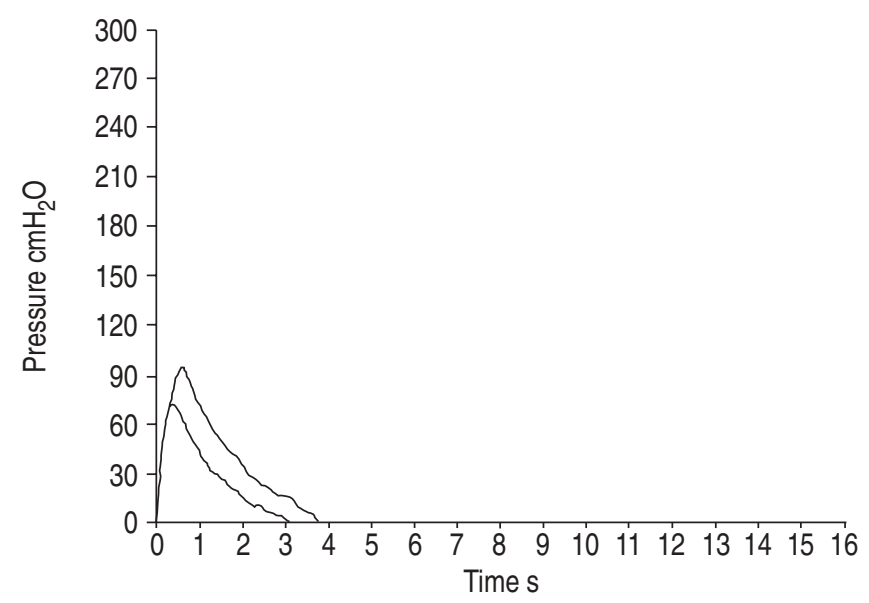

Fig. 1.-A sustained maximum inspiratory pressure curve.

carried out repeated inspiratory manoeuvres based on this template, which they attempted to match on each breath. Each SMIP was expressed as Joules [21, 22]. This phase consisted of a maximum of up to 36 inspiratory manoeuvres in groups of six efforts, as described in the test of incremental respiratory endurance $[21,22]$. The patient had to achieve $90 \%$ of the template on any given breath to continue with the series. The duration of treatment varied between patients, depending upon each subject's time to the point of failure. Hence, each group of six inspiratory efforts was characterised by a shorter rest interval between each inspiratory manoeuvre. The rest intervals reduced from 1 min to 45, 30, 15, 10 and $5 \mathrm{~s}$. A leak calibration constant was calculated, from flow rate $(\mathrm{Q})$, as follows:

$$
\mathrm{Q}=3.226 \times 10^{-6} \times \sqrt{\mathrm{p}}
$$

where pressure (p) was expressed in $\mathrm{N} \cdot \mathrm{m}^{-2}$ and $\mathrm{Q}$ in $\mathrm{m}^{3} \cdot \mathrm{s}^{-1}$. Power (P; in watts) was then calculated as follows:

$$
\mathrm{P}=\mathrm{p} \times \mathrm{Q}
$$

The work per breath was derived from the power curve and expressed in Joules [22]. All patients were familiar with the RT2 device and the RIM protocol.

Any sputum produced during either treatment was expectorated into a pre-weighed container. At the end of the treatment session, expectorated sputum was collected into the same container for a further $30 \mathrm{~min}$. The containers were coded and the sputum analysed by the laboratory staff unaware of the treatment group to which the patient had been assigned.

\section{Measurements}

Sputum weight was derived from the total weight minus the pre-use pot weight. The same balance (Ohaus Precision Advanced; Ohaus, Cottenham, Cambridge, UK) was used for all measurements. Sputum was refrigerated until processing, which comprised dilution 1:5 weight/weight with saline and 30 min mixing on a slow rotating mixer to liquefy the sample, which was then centrifuged at $10,000 \times g$ for $30 \mathrm{~min}$. The upper sol-phase was removed and stored at $-70^{\circ} \mathrm{C}$ until analysed.

Total protein concentration was determined by a modification of the micro-Lowry method, using albumin as a standard (P5656; Sigma, Dorset, UK) [5].

Human neutrophil elastase (HNE) was determined by a double antibody sandwich enzyme-linked immunosorbent assay (ELISA) developed for neutrophil elastase- $\alpha 1$-antiproteinase 
Table 1.-The forced expiratory volume in one second (FEV1), maximum inspiratory pressure (MIP) and sustained maximum inspiratory pressure (SMIP) at commencement of treatment in patients undergoing standard physiotherapy (SP) or resistive inspiratory manoeuvres (RIM)

\begin{tabular}{lcc}
\hline & SP days 1 \& 3, & RIM days 1 \& 3, \\
& RIM days 2 4 & SP days 2 \& 4 \\
\hline FEV1 \% pred & $48.3(37.6-59.0)$ & $59.7(40.1-79.3)$ \\
MIP cm ${ }_{2} \mathrm{O}$ & $132.2(111.8-153.0)$ & $132.4(97.4-167.4)$ \\
SMIP J & $9.8(6.8-12.8)$ & $9.6(6.1-13.1)$ \\
\hline
\end{tabular}

Data are presented as mean (95\% confidence interval).

complex and modified by using a mouse anti-human neutrophil elastase (Dako, Ely, UK) coating antibody [5].

IL-8 was determined by an in-house double antibody sandwich ELISA.

The laboratory researcher was blind to the treatment administered to patients.

FEV1, forced vital capacity $(\mathrm{FVC})$ and their ratio (FEV1/ FVC) were determined by spirometry and results were expressed as \% predicted [23]. Height, weight and skin-fold thickness were determined, and fat free mass (FFM) was calculated [21]. A low FFM was defined as being less than the lower 5th centile for local healthy subjects of matched age and sex [24].

\section{Statistical analysis}

Data were not normally distributed and were $\log _{10}$ transformed for analysis by t-test. Data are presented as geometric mean and 95\% confidence intervals. A pairedsample t-test was used to compare the concentrations of protein, IL-8 and HNE between the two types of treatment and between the two sessions of the same type of treatment (i.e. RIM or SP). Spearman's rank correlation test and linear regression were used to determine relationships between variables.

\section{Results}

\section{Patients}

The FEV1, MIP and SMIP were similar in the patients commencing treatment with SP or RIM (table 1). The proportion of patients with a normal or low FFM was also similar between the treatment groups (Chi-squared $\mathrm{p}=0.66$ ). Within the whole group, there were 11 patients with a low and nine with a normal FFM. The mean number of resistive inspiratory manoeuvres achieved was 24.6 (19.7-29.4; range 8-36). This reflected a treatment time between 10 and $40 \mathrm{~min}$, including rest periods, as described in the protocol. The number of resistive breaths was related to FEV1 $(r=0.83$, $\mathrm{p}<0.01)$.

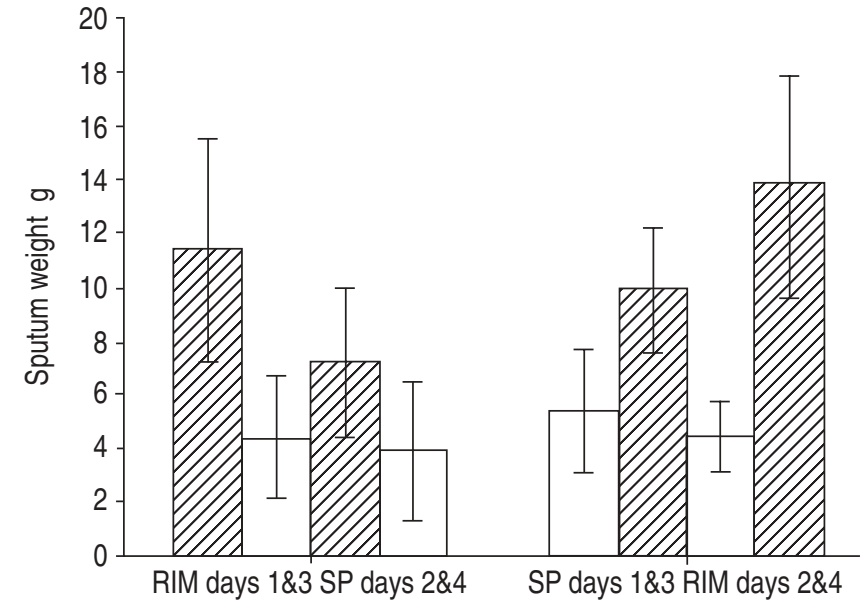

Fig. 2. - The mean weight of sputum expectorated during resistive inspiratory manoeuvres (RIM; $\mathbb{Q}$ ) treatment and standardised physiotherapy (SP; $\square$ ).

\section{Expectorated sputum}

The mean weight of sputum expectorated during RIM treatment was greater than for the SP and was not affected by the order in which the treatments were given (fig. 2). The pooled mean weight of sputum expectorated during and following SP was $4.9 \mathrm{~g}(3.31-6.56)$ compared with $10.0 \mathrm{~g}$ (7.65-12.37) for the RIM method.

The concentration of protein, IL-8 and HNE (per $g$ of sputum) was not different between the treatments or between days, nor was it affected by the order in which the treatments were given. The absolute amount of each component cleared was greater with the RIM method (table 2, fig. 3).

The mean difference between the absolute amounts of protein, IL-8 and HNE obtained after RIM and SP were $66.3 \mathrm{mg}(32.5-100.2), 4.6 \mathrm{ng}(-2.0-11.4)$ and $297.3 \mu \mathrm{g}$ (60.1-34.5), respectively.

The FFM, FEV1 and inspiratory muscle function (MIP and SMIP) were related, (all $\mathrm{p}<0.001)$. No difference was found between patients with a low or normal FFM for the clearance of any sputum component studied. For the whole group, FFM or FEV1 had no influence on the amount or concentration of any sputum components produced during SP or RIM.

\section{Discussion}

This short-term study, carried out during the first 4 days of antibiotic treatment for worsening respiratory symptoms, demonstrated that repeated inspiratory manoeuvres against a fixed resistance, standardised by visual bio-feedback to produce single-breath sustained inspiratory pressures of $80 \%$ of maximum, increased the wet weight of sputum expectorated over a 60 -min period compared with SP. The increased quantity of expectorated sputum enhanced the clearance of

Table 2.-Clearance of protein, interleukin (IL)-8 and human neutrophil elastase (HNE) in patients undergoing standard physiotherapy (SP) or resistive inspiratory manoeuvres (RIM)

\begin{tabular}{lllr}
\hline & Total protein $\mathrm{mg}$ & \multicolumn{1}{c}{ IL-8 ng } & HNE $\mu \mathrm{g}$ \\
\hline SP & $41.6(19.5-89.1)$ & $1.02(0.45-2.24)$ & $138.0(54.9-346.7)$ \\
RIM & $97.7(63.1-154.9)^{* *}$ & $1.47(1.22-1.78)^{* *}$ & $338.8(119.5-562.3)^{*}$ \\
\hline
\end{tabular}

Data are presented as geometric means $(95 \%$ confidence interval). *: $\mathrm{p}<0.05 ; * * \mathrm{p}<0.01$. 


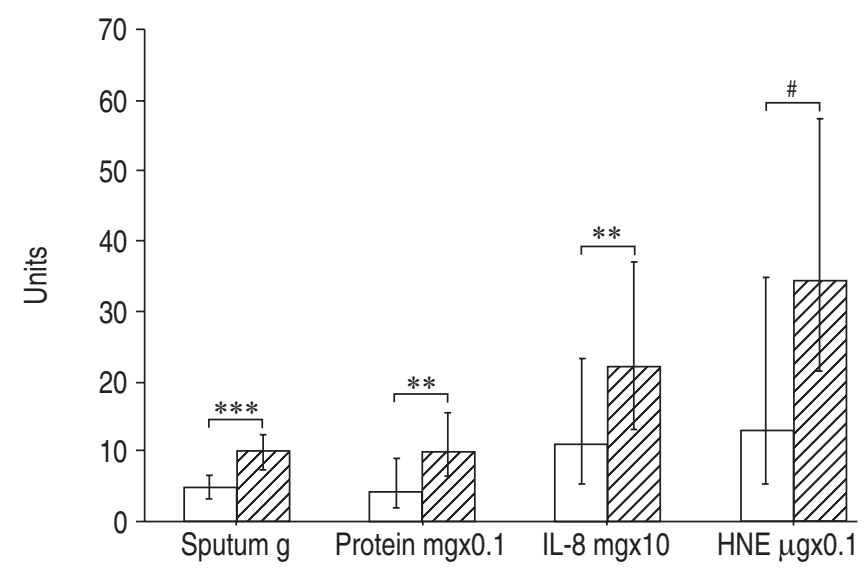

Fig. 3.-Clearance of protein, interleukin (IL)-8 and human neutrophil elastase (HNE) in sputum from patients undergoing standard physiotherapy (SP; $\square$ ) or resistive inspiratory manoeuvres (RIM; $\mathbb{Z}$ ). : $\mathrm{p}<0.05 ; * *: \mathrm{p}<0.01 ; * * *: \mathrm{p}<0.001$.

protein, IL-8 and immunoreactive HNE from the airways. The design of the study, with random allocation to the order of treatments, allowed the authors to demonstrate that RIM increased the weight of expectorated sputum irrespective of the order of treatments or the day of treatment.

An objective of physiotherapy is to increase expiratory flow rates with resultant high shearing forces, facilitating sputum mobilisation and transfer to more central airways. Hence, treatments usually incorporate techniques to avoid dynamic compression or collapse of airways caused by raised transthoracic pressure, and the resultant distal movement of the equal pressure point during forced expiration and coughing [25-27]. The finding in this study that RIM led to increased expectoration of sputum requires explanation, although the underlying mechanism in this study was not examined. The increased weight of expectorated sputum suggests the possible mobilisation and clearance of more peripherally situated secretions by RIM than that achieved by SP. This may be due to the effect of sustained near maximal inspiratory efforts on air and fluid dynamics in the distal airways. Within a distensible tube, such as the smaller airways, flow is proportional to pressure, and the volume of fluid or air moved depends on the duration over which the driving pressure is exerted [28]. The RIM method requires the patient to carry out a full inspiratory vital capacity manoeuvre at $80 \%$ of maximal pressure, by breathing against a fixed resistance. This leads to an extended inspiratory time secondary to the reduced airflow at the mouth. Thus, RIM may increase inspiratory airflow to more peripheral airways. If this occurs, there may be shearing forces acting on airways secretions, causing the break up of sputum during the inspiratory phase, which might reduce the need for higher expiratory flow rates and delay the time to reach the equal pressure point in small airways. The RIM method may also move the equal pressure point proximally by reducing $\mathrm{RV}$ and enabling increased airflow with less dynamic compression from a starting point of lower lung volume [29]. It may be of note that the patients at this centre were familiar with the active cycle of breathing technique, the drainage and percussion techniques and the RIM. Skill acquisition of both techniques is likely to be a factor in determining the effectiveness of treatment.

Sputum weight rather than volume was determined in this study, as expectorated sputum tended to be inhomogeneous in nature. As physiotherapy may produce benefits through removal of injurious products from the airways, a range of indicators of the pulmonary inflammatory response to chronic bacterial infection was also determined. The RIM treatment produced greater clearance of total protein, a general indicator of increased microvascular exudation and cellular breakdown in the airways and cause of increased sputum viscosity. The increased clearance of HNE, a measure of the neutrophil load in the airways, indicates the potential for removal of injurious products. This may be added to by the greater clearance of IL-8, a potent chemo-attractant for neutrophils, during and after the RIM treatments [30]. These comments are speculative and the demonstration of any longterm benefit from such effects requires further studies.

As the lung disease progresses, airways instability leading to collapse may confound the effectiveness of standardised physiotherapy and other techniques may be needed at different stages of the disease to achieve effective sputum expectoration. The resistive inspiratory manoeuvres method may also have the advantage that its settings of pressure and time are defined by the impaired pulmonary dynamics of the patient on each occasion it is used. It would be possible to adapt the software used in this method to enable patients to accurately breathe from a known lung volume, as required by protocols such as the active cycle of breathing techniques or autogenic drainage. Further studies are required to investigate such possibilities. At present, the authors have demonstrated that a series of near maximal inspiratory efforts against a fixed resistance appears to be more effective, in single treatment episodes, than standardised physiotherapy for sputum for expectoration in patients in a respiratory exacerbation, and it leads to enhanced clearance of inflammatory and injury mediators.

\section{References}

1. Davies PB, Drumm M, Konstan MW. Cystic fibrosis: state of the art. Am J Respir Crit Care Med 1996; 154: 1229-1256.

2. Smith JJ, Travis SM, Greenberg EP, Welsh MJ. Cystic fibrosis airway epithelia fail to kill bacteria because of abnormal airway surface fluid. Cell 1996; 85: 229-236.

3. Matsui H, Grubb BR, Tarran R, et al. Evidence for pericilliary liquid layer depletion, not abnormal ion composition, in the pathogenesis of cystic fibrosis. Cell 1998; 95: 1005-1015.

4. Konstan MW, Hilliard KA, Norvell TM, Berger M. Bronchoalveolar lavage findings in cystic fibrosis patients with stable clinically mild lung disease suggest ongoing infection and inflammation. Am $J$ Respir Crit Care Med 1994; 150: 448-454.

5. Nixon LS, Yung B, Bell SC, Elborn JS, Shale DJ. Circulating immunoreactive interleukin-6 (IL-6) in cystic fibrosis. Am J Respir Crit Care Med 1998; 157: 1764-1770.

6. Konstan MW, Berger M. Current understanding of the inflammatory process in cystic fibrosis: onset and etiology. Pediatr Pulmonol 1997; 24: 137-142.

7. Dean TP, Dai Y, Shute JK, Church MK, Warner JO. Interleukin-8 concentrations are elevated in bronchoalveolar lavage, sputum and sera of children with cystic fibrosis. Pediatr Res 1993; 34: 159-161.

8. Sagel SD, Kapsner R, Osberg I, Sontag MK, Accurso FJ. Airway inflammation in children with cystic fibrosis and healthy children assessed by sputum induction. Am J Respir Crit Care Med 2001; 15: 164: 1425-1431.

9. Sagel SD, Sontag MK, Wagener JS, Kapsner RK, Osberg I, Accurso FJ. Induced sputum inflammatory measures correlate with lung function in children with cystic fibrosis. $J$ Pediatr 2002; 141: 811-817.

10. Schulz C, Wolf K, Harth M, et al. Expression and release of interleukin-8 by human bronchial epithelial cells from patients with chronic obstructive pulmonary disease, smokers and never-smokers. Respiration 2003; 70: 254-261.

11. Beeh KM, Kornmann O, Buhl R, Culpitt SV, Giembycz MA, 
Barnes PJ. Neutrophil chemotactic activity of sputum from patients with COPD: role of interleukin 8 and leukotriene B4. Chest 2003; 123: 1240-1247.

12. Nelson R, Miller S, Hall D, Clayton B. Chest physiotherapy in cystic fibrosis. Thorax 1995; 50: 1123-1124.

13. Williams MT. Chest Physiotherapy and cystic fibrosis: why is the most effective form of treatment still unclear? Chest 1994; 106: 1872-1882.

14. Webber BA, Pryor JA. Physiotherapy techniques. In: Pryor JA, Webber BA, eds. Physiotherapy for Respiratory and Cardiac Problems, 2nd Ed. Edinburgh, Churchill Livingstone, 1998; pp.137-155.

15. Thomas J, Cook DJ, Brooks D. Chest physical therapy management of patients with cystic fibrosis: a meta-analysis. Am J Respir Crit Care Med 1995; 151: 846-850.

16. Pryor JA, Webber BA, Hodson ME, Batten JC. Evaluation of the forced expiration technique as an adjunct to postural drainage in cystic fibrosis. BMJ 1979; 2: 417-418.

17. Lannefors L, Wollmer P. Mucus clearance with three chest physiotherapy regimens in cystic fibrosis: a comparison between postural drainage, PEP and physical exercise. Eur Respir J 1992; 5: 748-753.

18. Apps EM, Kieselman R, Reinhardt D, et al. Sputum rheology changes in cystic fibrosis lung disease following two different types of physiotherapy: flutter vs autogenic drainage. Chest 1998; 114: 171-177.

19. Abbott J, Dodds M, Webb AK. Health perceptions and treatment adherence in adults with cystic fibrosis. Thorax 1996; 51: 1233-1238.

20. Kettler LJ, SM Sawyer, HR Winefield, HW Greville.
Determinants of adherence in adults with cystic fibrosis Thorax 2002; 57: 459-464.

21. Ionescu AA, Chatham K, Davies CA, Nixon LS, Enright S Shale DJ. Inspiratory muscle function and body composition in cystic fibrosis. Am J Respir Crit Care Med 1998; 158: 1271-1276.

22. Chatham K, Baldwin J, Griffiths H, Summers L, Enright S. Inspiratory muscle training improves shuttle run performance in healthy subjects. Physiotherapy 1999; 85: 676-683.

23. Guidelines for the measurement of respiratory function. Recommendations of the British Thoracic Society and the Association of Respiratory Technicians and Physiologists. Respir Med 1994; 88: 165-195.

24. Office of population censuses and surveys. The Dietary and Nutritional Survey of British Adults 1990: 237.

25. Van der Schans CP. Forced expiratory manoeuvres to increase transport of bronchial mucus: a mechanistic approach. Mondaldi Arch Chest Dis 1997; 52: 367-370.

26. Zach MS. The physiology of forced expiration. Paediatr Respir Rev 2000; 1: 36-39.

27. Pryor JA. Physiotherapy for airways clearance in adults. Eur Respir J 1999; 14: 1418-1424.

28. Regnis JA, Donnelly PM, Robinson M, Alison JA, Bye PT. Ventilatory mechanics at rest and during exercise in patients with cystic fibrosis. Am J Respir Crit Care Med 1996; 154: 1418-1425.

29. Maclem PT. The mechanics of breathing. Am J Respir Crit Care Med 1998; 157: 588-594.

30. Bonfield TL, Panuska JR, Konstan MW, et al. Inflammatory cytokines in cystic fibrosis lungs. Am J Respir Crit Care Med 1995; 152: 2111-2118. 